\title{
System Organization of the Relationship Between Emotional Stress and Sleep
}

\section{Yumatov EA and Pertsov SS}

\author{
P. K. Anokhin Research Institute of Normal Physiology, National Research University, Moscow Power Engineering Institute, Moscow, Russia
}

Corresponding author: Yumatov Evgeny A. P.K. Anokhin Research Institute of Normal Physiology, National Research University, Moscow Power Engineering Institute, Moscow, Russia, Tel: 89036149343; E-mail: eayumatov@mail.ru.

Received: Sep 06, 2016; Accepted: Sep 26, 2016; Published: Sep 30, 2016

Citation: Yumatov EA, Pertsov SS. System Organization of the Relationship Between Emotional Stress and Sleep. J Neurol Neurosci. $2016,7: 5$.

\section{Abstract}

Here we review an urgent medico-social problem exists, which integrates the social environment, psychoemotional state of people, and sleep disorders. A close interrelation exists between the human psycho-emotional state and sleep. Emotional stress is followed by sleep disorders, while sleep deficiency increases the severity of stress.

Experimental data illustrate the mechanisms for a relationship between emotional stress and sleep at various levels of organization, including the structuraland-neurophysiological, neurochemical, and somatic-andautonomic levels.

The results of studies performed by Russian and foreign researchers allowed us to make the principal conclusions on a relationship between the emotional state and sleep. These data show the existence of common neurophysiological mechanisms and antagonistic relations of neurochemical processes in emotional stress and sleep.

Keywords: Emotional stress; Sleep; Wakefulness; Neurophysiology; Somnology; Insomnia

\section{Introduction}

An urgent medico-social problem exists, which integrates the social environment, psycho-emotional state of people, and sleep disorders.

Emotional stress results from hard work, irrational organization of the rest period, social-and- domestic problems, environmental and technogenic accidents, numerous social conflicts, etc. $[1,2]$.

The adverse effects of stress are manifested in the development of psychoneurotic diseases, depressive states, and sleep disorders and increase in the incidence of alcoholism and drug abuse [3-5].

A close relationship exists between human psychoemotional state and sleep [6-8]. A normal physiological sleep is the major anti-stress factor, which determines the working capacity, fatigability, etc. By contrast, sleep deprivation contributes to the development of emotional stress, which is as a major cause of sleep disorders. The following vicious circle is formed: stress-induced disturbances in sleep serve as an additional provoking factor for the increased severity of stress.

A physiological role of wakefulness and sleep is determined by different biological expediency of these states. The emotional state in wakefulness characterizes an active goalseeking behavior of the subject, which is directed toward the satisfaction of vital social and biological requirements. In fact, sleep reflects the state of relaxation and termination of a goalseeking behavioral activity. In whole, wakefulness and sleep are in reciprocal relationships. An increase in the degree and prolongation of negative emotions are followed by emotional changes in the brain activity and progression of emotional strain, which violates a normal sleep.

A biological interrelation exists between emotions and sleep. Therefore, a question arises regarding the similarity and differences in certain mechanisms that combine the emotional strain and sleep at various levels of organization (structuraland-neurophysiological, neurochemical, and somatic and autonomic).

\section{Somatic and autonomic disorders in emotional stress}

$H$. Selye was the first to define the notions of stress as a general nonspecific adaptation syndrome of the body [9]. Stress is the reaction of strain, which progresses as a nonspecific body response to the influence of extreme, unfavorable environmental factors, or "stressors". They include pathogenic agents, toxic and foreign substances, physical load, and other inadequate factors.

The researches by P.K. Anokhin, K.V. Sudakov, and E.A. Yumatov showed that long-term negative emotions cause emotional stress. This state develops in a conflict behavioral situation, when any subject cannot satisfy the dominant requirement for a long time $[1,2,5]$.

Modern medical-and-biological and psychophysiological studies illustrate that emotional stress has a variety of effects on the body functions $[10,11]$. Emotional stress serves as a cause of many psychosomatic diseases (psychoses, neuroses, cardiovascular diseases, and ulcerative-and-atrophic injuries to the gastrointestinal tract); immune dysfunction and increased 
predisposition to viral and infectious diseases; autoimmune processes; rheumatic diseases and osteochondrosis; oncological diseases; hormonal disturbances, sexual dysfunction, and sleep disorders; etc. Stress affects the genetic apparatus of cells, which contributes to congenital developmental defects and child diseases.

Emotional stress is primarily formed as a central neurogenic process. All peripheral manifestations of abnormalities in vital somatic-and-autonomic functions are the secondary process, which results from emotional over excitation [2].

The sympathetic and parasympathetic parts of the autonomic nervous system are involved in emotional reactions. An individual relationship between these compartments determines the type of autonomic signs for qualitatively different emotional reactions.

The realization of emotional excitations is also mediated by the hormonal mechanisms (primarily, by the pituitary-adrenal system). Emotional excitation is characterized by the following hormonal changes: increase in blood catecholamine concentration (epinephrine, norepinephrine, and dopamine) due to their release from the adrenal glands; acceleration of secretion of thyroid hormones; and elevation of the contents of cyclic AMP, prostaglandins, and renin in blood plasma. The elevation of plasma corticosterone level is typical of emotional stress and progresses with an increase in the degree of stress.

In accordance with the stages of stress described by $\mathrm{H}$. Selye, hormonal reactions in emotional stress develop in several phases. An initial increase in blood catecholamine level is accompanied by a decrease in their concentration in the adrenal glands. These changes are followed by activation of the adrenocorticotropic function and increase in catecholamine synthesis. The concentration of corticosterone in blood plasma decreases under conditions of long-term stress exposures. An increase in the amount of catecholamines and corticoids causes hyperglycemia and elevation of glycoprotein content and blood cholesterol.

These humoral and hormonal reactions are triggered due to activation of the hypothalamic-pituitary-adrenocorticotropic mechanisms [2]. The nervous and humoral influences in emotional stress are followed by a generalized reaction of autonomic functions.

Emotional reactions due to stimulation of the ventromedial hypothalamus are characterized by several somatic-andautonomic manifestations, including a change in the depth and rate of respiration; increase in the heart rate (HR), blood pressure, and cardiac output; defecation and urination; elevation of corticosteroid concentration in blood plasma; constriction of the intestinal, renal, and cavernous vessels; and significant dilation of skeletal muscle vessels.

The cardiovascular response is manifested in blood pressure changes and redistribution of the circulating blood. Emotional stress is accompanied by an increase in blood supply to the brain, heart, and lungs and decrease in the blood flow rate in abdominal and pelvic organs. A significant pressor response is observed under conditions of emotional strain due to short- term or long-term electrical stimulation of negative emotiogenic structures in the hypothalamus $[2,10,12]$.

Persistent hypertension develops due to the vicious circle, which is characterized by primary emotional excitation in the limbic and reticular structures of the brain (activating the hormonal function of the adrenal glands) and secondary feedback effect of adrenal hormones on the central adrenergic mechanisms in the midbrain reticular formation $[2,13]$.

The hypertensive reaction to stimulation of negative emotiogenic structures in the hypothalamus is related to hypofunction of the baroreceptor sinocarotid and aortic reflexogenic mechanisms. The hypertensive reaction during emotional overload can result from an increase in the cardiac output and total peripheral vascular resistance.

An increase in the peripheral vascular resistance is associated not only with the nervous influences, but also with the humoral and hormonal mechanisms. They include the renin-angiotensin system, which determines a hypertensive effect of blood renin and involvement of this agent in enzymatic reactions. These processes are followed by the appearance of angiotensinogen-2 in blood plasma, which has a strong vasoactive effect.

Several humoral factors, which determine the elevation of blood pressure under conditions of emotional stress, also produce a hypertensive effect. These factors are presented by epinephrine and corticoids (released from the adrenal glands to the blood during emotional stress), as well as by hormones of the hypophysis and thyroid gland (vasopressin and thyrotropin). Emotional stress is accompanied by a change in the sensitivity of beta-adrenoceptors, which also contributes to the pressor reaction.

Much attention was paid to the specific features of heart function during emotional reactions and stress $[4,10]$. Most natural, negative emotional reactions, which are accompanied by an aggressive and active defensive behavior or mental emotional strain, cause an increase in HR. An increase in HR is often related to activation of the sympathoadrenal system. Sometimes, these changes are associated with a decrease in the tone of vagal nuclei.

Emotional overload can cause cardiac dysfunction $[2,4]$. Stimulation of the ventromedial hypothalamic nucleus is accompanied by a change of the $P$ and $T$ waves in ECG, which illustrates the impairment of coronary blood supply. Ventricular extrasystoles are observed under these conditions. In these experiments, some animals died from acute heart failure, vascular insufficiency, and ventricular fibrillation. Histologically, the myocardium in animals exposed to longterm stimulation of the hypothalamic emotiogenic zones was characterized by degradation of mitochondria, damage to myofibrils, vacuolar-granular degeneration, increase in the number of lipoprotein granules and lysosomes, and loosening of the chromatin in cell nuclei. These changes are typical of acute myocardial ischemia and pre-infarction state.

Cardiac abnormalities in emotional strain are related to an excessive effect of catecholamines that appear during 
hyperactivation of the sympathoadrenal mechanism. Stimulation of the hypothalamic emotiogenic zones in rabbits was accompanied by an increase in the content of glucocorticoids and epinephrine and decrease in the level of norepinephrine in the myocardium. Similar changes were revealed during emotional-pain stress and restraint stress in rats.

The sympathoadrenal influences contribute to atrial flutter, fibrillation, and myocardial necrosis in animals during experimental emotional stress. The cholinergic parasympathetic influences on the heart have a protective role and prevent cardiovascular disorders.

Negative emotions due to a passive defensive behavior, depression, and fear can be accompanied by activation of the parasympathetic autonomic nervous system, which results in bradycardia (as shown in humans, monkeys, dogs, and cats).

Emotional stress of the hypothalamic origin causes a decrease in the activity of $\mathrm{Na+} / \mathrm{K}+-$ ATPase (enzyme for the active transport of ions in myocardial cell membranes), which is usually accompanied by the reduction of mitochondrial phosphorylation activity. Emotional-pain stress and restraint stress in rats were also accompanied by the inhibition of respiration and oxidative phosphorylation in myocardial mitochondria, as well as by a decrease in the membrane potential of myocardial fibers and $\mathrm{Na}+/ \mathrm{K}+-$ ATPase activity. These abnormalities can serve as a cause of the reduced excitability in cardiac cells, which is mainly related to the impairment of $\mathrm{Na}+/ \mathrm{K}+$ transport across the cell membranes.

The pathogenesis of cardiac dysfunction during emotional strain includes hypoxia and activation of lipid peroxidation in the myocardium. This conclusion was made from the results of experiments on rats subjected to immobilization and emotional-pain stress.

A change in the concentration of glucocorticoids and catecholamines promotes the development of hypoxia. These changes are accompanied by the inhibition of energy processes in the myocardium, activation of lipid peroxidation in membranes, damage to membranes by peroxides and proteolytic enzymes, and impairment of ion transport across the cell membranes. These abnormalities are followed by necrotic lesions, reduction or loss of the cell excitability, and development of cardiac dysfunction.

Gastric ulceration is one of the major signs of emotional stress. Emotional stress-induced ulceration is primarily related to the sympathetic and parasympathetic influences and effect of endogenous humoral and hormonal factors.

Emotional overstrain is one of the factors that determines renal dysfunction and destructive processes in the liver parenchyma.

Breathing is also involved in the emotional reactions. Emotional stress is accompanied by the increase in pulmonary ventilation and oxygen consumption.

Emotional stress causes a variety of blood changes, including the degranulation of basophils, thrombocytopenia, neutrophilic leukocytosis, and decrease in the count of mature granulocytes in the bone marrow.

Emotional stress is followed by a change in immune reactivity and pain threshold. Stress-induced analgesia is a typical reaction, which results in an increase of the pain threshold (for example, to electrocutaneous stimulation).

Besides somatic-and-autonomic manifestations, emotional stress can cause serious neurotic reactions and behavioral inadequacy $[14,15]$. The subjects with these changes cannot rapidly make a correct decision. They are characterized by excessive emotionality in response to irrelevant events, overanxiousness, anxiety, suspiciousness, and tendency to longlasting emotional sufferings. Emotional reactions lose their plasticity and cannot serve as a reliable factor to mobilize the behavior to achieve a certain adaptive result. Sometimes, neurotic patients cannot perform a particular action to eliminate the unfavorable factor. Emotional overstrain in animals causes a complex of the following neurotic symptoms: impairment of conditioned behavior, reduction of the learning capacity, sleep disorders, etc.

These data indicate that emotional stress is accompanied by a complex of nonspecific reactions, which develop in any conflict situation and concern the vital physiological functions. It results in disintegration of various functional systems in the body during wakefulness and sleep.

\section{Characteristics and disturbances of sleep due to emotional stress}

A large body of evidence shows the interrelation between psycho-emotional (characteristic) state of an individual, level of emotional strain, sleep-wakefulness cycle, quality of nocturnal sleep, and phase structure of nocturnal sleep $[6,16]$.

A normal physiological sleep has several consecutive phases. Each phase plays a particular biological role and is manifested in specific features of EEG, eye movements, muscle tone, and change in some autonomic functions $[17,18]$.

Sleep is not a homogenous process. In humans and animals, sleep occurs in various stages of non-rapid eye movement sleep (NREM or non-REM sleep) and rapid eye movement sleep (REM sleep). Human nocturnal sleep consists of 4-5 cycles of NREM sleep and REM sleep. The length of a whole sleep cycle is relatively constant (90-100 $\mathrm{min}$ in healthy people). The percentages of NREM sleep and REM sleep are $80-75$ and $20-25 \%$, respectively.

NREM sleep has the following characteristics.

1. Stage A: characterizes the transition from relaxation to drowsiness. Alpha waves are present in EEG. This stage can be considered as a manifestation of sleep motivation.

2. Stage B: reflects the state of drowsiness. EEG is characterized by the decrease in alpha waves and presence of theta waves, beta waves, and individual delta waves. Slow eye movements can be found in the electrooculogram (EOG) during stages $A$ and $B$. 
3. State C: illustrates the state of light sleep. EEG includes sleep spindles (frequency 14-16 oscillations per sec, amplitude 30-50 $\mu \mathrm{V}$ ). K-complexes (two-to-three-phase waves of 0.5-1 sec) can be also observed. This state is accompanied by lowamplitude slow oscillations in the delta and theta range and mild beta rhythm. Slow eye movements are reduced or disappear (as confirmed by the recording of EOG). Electromyography (EMG) demonstrates a decrease in the amplitude of muscle biopotentials.

4. Stage D: characterizes sleep of intermediate depth. The EEG contains high-amplitude delta waves and sleep spindles.

5. Stage E: reflects deep sleep. The EEG is characterized by a predominance of high-amplitude slow delta waves and disappearance of sleep spindles and K-complexes.

NREM sleep (deep sleep) results in the normalization of homeostasis (violated after many-hours-long wakefulness), decrease in the respiratory rate and $H R$, reduction of blood pressure and body temperature, muscle relaxation, and slow eye movement. The total number of movements in a sleeping individual becomes lowest with an increase in the depth of NREM sleep.

American researches E. Azerinsky and N. Kleitman discovered the phenomenon of REM sleep. During this stage, NREM sleep is interrupted by the periods of low-amplitude high-frequency EEG (30-40 oscillations per sec).

\section{The stage of REM sleep can be characterized as follows:}

1. No activity of the facial and neck muscles (the tone of other muscles practically does not differ from that in deep stages of NREM sleep);

2. Appearance of rapid eye movements (REM stage) of 0.5-1.5 sec in duration;

3. Presence of the wakefulness rhythm (beta waves) in EEG;

4. Pronounced fluctuations in autonomic indexes; and

5. Presence of dreams.

Sleep deprivation is first followed by an increase in the duration of low-wave delta sleep $[19,20]$. Long-term insomnia causes emotional stress with severe neurotic disorders. Selective deprivation of REM sleep is followed by a change in the brain mental activity (e.g., increase in emotional irritability). Hallucinations and paranoid thoughts can be observed with an increase in the severity of this state.

An extensively used, standard methodology of somnology is directed toward studying the sleep of patients in ambulatory and clinical environments, but does not allow us to perform a control of human sleep under real, everyday home conditions.

The information system was developed, which allows monitoring of human physiological functions during sleep in a real everyday life. This system provides the following issues: recording, identification, and analysis of the phase structure of sleep; awakening of an individual in the certain time and predetermined optimal phase of sleep (as regards the psychophysiological state of this individual); interruption of a hazardous phase of sleep; monitoring of cardiac activity and breathing; cross-correlation analysis of HR and respiratory rate (reflecting the degree of emotional strain [21]; and viewing and analyzing the results on a personal computer $[22,23]$.

A high level of emotional strain during wakefulness and severe emotional sufferings cause insomnia (sleep disorder), which is manifested in a decrease in the quality of sleep, sleeplessness, difficulties in falling asleep, numerous awakenings in the nighttime, early morning awakening, sleepiness and fatigue after sleep, and excessive movements and snoring during sleep.

\section{Neurophysiological mechanisms of emotional stress and sleep}

Emotional stress is primarily formed as a central neurogenic process. All peripheral manifestations of abnormalities in vital somatic-and-autonomic functions are the secondary process, which results from emotional over-excitation.

Emotional excitation is initially formed in the hypothalamic, limbic, and reticular structures of the brain. Emotional excitation is characterized by generalized spreading to the brain cortex via ascending pathways. Moreover, emotional excitation descends, involves the autonomic nervous system, and stimulates the hormonal pituitary-adrenal activity to realize somatic-and-autonomic and behavioral manifestations of emotional reactions [2].

Limbic structures of the brain play a role in the formation of emotional reactions. They include the paleocortex and archicortex, some fields of the cerebral neocortex (orbital areas and temporal regions), most part of the diencephalon, and reticular formation of the midbrain.

Among a variety of central structures, the hypothalamus has a triggering role in the formation of motivations and emotional excitation. This structure has a particular sensitivity to humoral factors, which are associated with various biological requirements. The hypothalamus is a major triggering zone of the autonomic and hormonal activity, which induces a motivated emotional behavior for avoidance and selfstimulation.

The studies by P.V. Simonov demonstrated a major role of four brain structures in the organization of emotional reactions (anterior regions of the cerebral neocortex, hippocampus, amygdala nuclei, and hypothalamus). The frontal neocortex is required for probabilistic forecasting of external events and evaluation of the possibility to satisfy a requirement. High-probability events can be identified due to activity of the frontal cortex [24].

Hippocampal function is associated with the detection of signals from low-probability events under conditions of uncertainty, when the level of emotional strain increases.

A particular function of the amygdaloid complex is to identify the dominant requirement, which should be satisfied first of all.

The hypothalamus plays a major role in the formation of various biological motivations due to metabolic requirements. 
The ventromedial hypothalamic nucleus has a specific role in the development of negative emotional reactions. Animal experiments showed that local electrostimulation of the ventromedial hypothalamus causes a complex of aggressive behavioral reactions, which are followed by severe hypertension.

Emotional reactions of the hypothalamic origin are primarily accompanied by a change of bioelectric activity in emotiogenic structures of the brain. For example, this state is characterized by an increase of theta waves in the hippocampus, as well as by desynchronization in the cerebellar amygdala and cerebral cortex.

A continuous stimulation of the hypothalamic emotiogenic zones for several hours was accompanied by significant phase changes of electrical activity in the cortical and subcortical structures. The majority of animals exhibited primarily a lowamplitude, high-frequency desynchronized activity in cortex leads over the 1st hour of stimulation. A regular "rhythm of tension" was recorded in the following subcortical structures: dorsal hippocampus, septum, amygdaloid complex, and reticular formation of the midbrain. During stimulation, some animals were characterized by the appearance of generalized, slow, spiked high-amplitude waves (amplitude $200 \mu \mathrm{V}$ ). Elevation of the respiratory rate, tachycardia, and increase in the muscle tone were observed at this stage of hypothalamic stimulation. Defecation and urination were revealed in many animals. Blood pressure was shown to increase and remained high for 20-40 $\min [2]$.

Stimulation of the midbrain reticular formation also caused a negative emotional state, which was accompanied by the hypertensive reaction. By contrast, bilateral coagulation of the reticular formation or transection of the midbrain prevented the development of arterial hypertension of the hypothalamic, renal, and baroreceptor origin and abolished the pituitaryadrenal response to stress. Blockade of the mesencephalic reticular formation by anodic polarization was followed by a shortening of the pressor response to hypothalamic stimulation.

The midbrain reticular formation is one of the major structures, which plays a role (in addition to the hypothalamus) in the regulation of blood flow. A close relationship exists between the hypothalamus, midbrain reticular formation, and limbic structures, which provides long-term reverberation of excitations between the brain structures.

Nonspecific medial regions of the thalamus are morphologically and functionally related to the reticular formation, hypothalamus, limbic structures, and neocortex. The medial thalamus is involved in processing and transfer of ascending activating influences from the reticular formation and hypothalamus to the higher brain regions. The ascending activating influences are a major component of the motivational and emotional excitation. The nonspecific thalamic regions have a particular role in the involvement of adrenergic hypothalamic substrates and reticular formation in the maintenance of emotional states.
Due to descending influences, the cerebral cortex can improve or diminish the manifestations of subcortical emotional reactions.

Therefore, individual structures of the brain do not serve as a center of certain emotional reactions. By contrast, the interaction between these structures contributes to the formation of emotional states. The central emotiogenic structures of the brain induce emotional reactions. It should be emphasized that emotional excitation involves a complex of limbic and reticular structures, which determines the development of somatic-and-autonomic manifestations of emotional stress.

Summation of the negative emotional reactions forms a basis for "stagnant emotional excitations". A long-lasting negative emotional state is associated with "persistent changes of cellular metabolism in the hypothalamus and brainstem" [1].

A wakeful state of the brain is provided by the reticular formation, which triggers the cortical mechanisms for wakefulness $[25,26]$. Electrical stimulation of the reticular formation during sleep is immediately followed by awakening of an animal. Destruction of the reticular formation or transection of the brainstem at the level of the midbrain causes sleep.

During wakefulness, sensory afferentation from the sense organs contributes to activation of the reticular formation that produces an ascending activating effect on the cerebral cortex. Deep sleep is observed during blockade of ascending activating influences on the cerebral cortex.

The limbic-and-hypothalamic and reticular structures of the brain are in reciprocal relationships. Excitation of the limbicand-hypothalamic structures is accompanied by suppression of the brainstem reticular formation, and vice versa.

Ascending activating influences of the reticular formation on the cerebral cortex are reduced with a decrease in the sensory inflow. Inhibitory influences of the frontal cortex on posterior hypothalamic neurons are diminished under these conditions. As a result, these cells produce a greater inhibitory effect on the brainstem reticular formation.

Somnogenic structures of the brain are involved in the formation of sleep behavior, as well as in synchronized and desynchronized phases of the brain activity [27-29].

NREM sleep is associated with activity of EEG-synchronizing structures in the brain [30]. Neurophysiological studies showed that NREM sleep is induced by the raphe nuclei. Local destruction of these nuclei is followed by chronic insomnia, which can result in death $[31,32]$.

Low-frequency stimulation of the solitary tract nucleus causes synchronization in EEG [26]. Falling asleep is accompanied the increase in neuronal activity in this zone. The EEG-synchronizing effect during stimulation of baroreceptors in the aorta and carotid sinus is realized via the solitary tract nucleus. 
Stimulation of the anterior hypothalamus is accompanied by the appearance of sleep spindles and slow-wave activity in EEG, which results from the interaction of the hypothalamus with the midbrain and thalamic structures [32,33].

Low-frequency stimulation of the head of the caudate nucleus is followed by the appearance of synchronizing cortical activity and behavioral signs of retardation [26,33].

Sleep and EEG synchronization are observed during electrical stimulation of the lateral preoptic area [34]. Destruction of this region causes insomnia, cachexia, and death of animals. W. P. Koella reported that the thalamus is a major synchronizing structure of the brain [35].

The orbital cortex plays a particular role in the mechanisms of synchronization. Extirpation of this structure is accompanied by the disappearance of sleep-related slow-wave activity.

A study of neurons in the orbital, parietal, and association cortex, lateral geniculate body, hippocampus, hypothalamus, thalamus, and reticular formation showed that neuronal activity in some brain structures significantly increases during sleep, which illustrates the absence of "generalized inhibition" in sleep [36].

Hence, stimulation of many brain structures can be followed by a synchronizing effect in EEG and behavioral signs of sleep. The thalamocortical system, which is responsible for synchronizing influences, serves as a major somnogenic structure. Other structures produce the regulatory effect on this system, which is mainly determined by activity of the humoral and physiological systems and effect of exogenous factors.

Paradoxical sleep is induced by the brain structures, which are localized in the upper regions of the caudal reticular nucleus and middle regions of the reticular nucleus in the pons Varolii and medulla oblongata [30,37-41]. They include the locus coeruleus, vestibular nuclei of the medulla oblongata, superior colliculus, and midbrain reticular formation. Local destruction of these areas was followed by the disappearance of REM sleep, but had little effect on NREM sleep and wakefulness.

These data indicate that the formation of emotional strain and sleep involves the same structures of the brain.

\section{Neurochemical mechanisms of emotional reactions and sleep}

Brain activity during wakefulness and sleep is mediated by various interrelated neurochemical mechanisms.

The central mechanisms of emotional reactions involve neurotransmitter processes (noradrenergic, serotoninergic, and dopaminergic) $[5,13]$. Negative emotional reactions and emotional stress are accompanied by specific changes in the metabolism of major transmitters (norepinephrine, acetylcholine, and serotonin) in emotiogenic structures of the brain. Previous studies revealed a change in the chemical sensitivity of neurons in the medial hypothalamus, medial thalamus, and midbrain reticular formation to neurotransmitters and neuropeptides.

A change in the chemical sensitivity of neurons is related to variations in the conformation of receptor proteins and functional activity of receptors [42].

The central mechanisms of emotional reactions are determined by selective reorganization of neurochemical properties and plastic reconstruction of the neurotransmitter metabolism in neurons of emotiogenic brain structures. It results in the formation of new neurotransmitter integration for emotional excitation, which determines the development of a negative emotional state and induces a complex of somatic-and-autonomic manifestations of emotional reactions $[5,13]$. By the neurochemical organization, neurotransmitter integration of the emotional state is a polychemical process.

Our studies revealed that the central mechanisms of emotional stress involve various endogenous peptides, including substance P, Delta Sleep-Inducing Peptide (DSIP), prolactin, etc. $[43,44]$.

Catecholaminergic processes play an important role in the neurochemical mechanisms of wakefulness. The ascending activating system of the brain, which determines wakefulness, is adrenergic. Urine excretion of epinephrine, norepinephrine, DOPA, and dopamine is highest in wakefulness, becomes minimum during NREM sleep, and occupies an intermediate position under conditions of REM sleep.

Many anti-sleep substances are chemically close to epinephrine or contribute to its accumulation in the nervous system. Phenamine-similar pharmaceuticals cause EEG desynchronization and lengthening of wakefulness. By contrast, phenothiazine products (Aminazin) with adrenolytic activity reduce the level and duration of wakefulness.

Various biologically active substances are involved in the neurochemical organization of sleep [45-47].

Serotoninergic substances, including L-tryptophan (serotonin precursor), contribute to a decrease in the onset latency and lengthening of REM sleep.

Monoamine oxidase inhibitors, which cause the accumulation of serotonin and norepinephrine in the brain, increase the length of NREM sleep and have an inhibitory effect on REM sleep [48].

The inhibition of serotonin synthesis in the brain causes insomnia, which can be prevented by treatment with 5hydroxytryptophan in intermediate doses (serotonin precursor). Serotonin antagonists, methysergide and Deseril, abolish the influence of tryptophan on the sleep structure. The prevention of sleep after treatment with $p$ chlorophenylalanine is related to the inhibition of tryptophan hydroxylase (enzyme for the biosynthesis of 5hydroxytryptamine). Administration of 5-hydroxytryptamine under these conditions has a normalizing effect on sleep. pChlorophenylalanine decreases the content of serotonin in the brain of rabbits and rats, which is accompanied by shortening of sleep (mainly due to the phase of NREM sleep). 
The raphe nuclei are responsible for slow-wave sleep and contain serotoninergic neurons. Administration of serotonin into the raphe nuclei contributes to the maintenance of NREM sleep [49].

Hallucinogenic agents (e.g., lysergic acid diethylamide) cause blockade of serotonin synapses and decrease the duration of REM sleep [50].

The content of a serotonin derivative, melatonin, in the pineal gland is characterized by circadian variations [51,52]. Sleep deprivation in healthy subjects is followed by the increase in 5-hydroxyindoleacetic acid excretion.

The influence of acetylcholine on the brainstem and mediobasal temporal cortex was shown to induce electroencephalographic and behavioral signs of sleep [53-55]. Physostigmine (eserine) with anticholinesterase activity increases the length of REM sleep [37]. Pilocarpine produces the same, but less significant effect. Deprivation of REM sleep for 4-5 days is followed by a selective decrease in the content of acetylcholine in rat brain. By contrast, the amount of acetylcholine increases after total sleep deprivation for 1 day.

The effects of gamma-aminobutyric acid (GABA) on sleep were studied in details. Experiments on cats showed that the rate of GABA release from a perforated surface of the cerebral cortex during sleep is threefold higher than in wakefulness. An intraperitoneal injection of GABA to mice causes short-lasting sleep. An intraperitoneal or intra-cerebroventricular infusion of GABA to cats is accompanied by shortening of REM sleep, but lengthening of NREM sleep. Administration of sodium butyrate (GABA-like substance) at low and high doses contributes to the onset of NREM sleep and REM sleep respectively [56]. Many products, including barbiturates, tranquilizers, and alcohol, have a suppressive effect on the phase of REM sleep.

Peptides play a role in the neurochemical mechanisms of sleep. DSIP was isolated by M. Monnier in 1974 . This peptide is present in the pineal gland, hypothalamus, and limbic structures of the brain and induces slow-wave sleep. Intracerebroventricular administration of vasoactive intestinal peptide has a stimulatory effect on REM sleep. Some endogenous peptides (endorphin, enkephalins, and dynorphin) are involved in the formation of emotional states during wakefulness and slow-wave sleep. Prostaglandin D2 is synthesized in the brain and modulates the activity of adenosinergic neurons $[57,58]$.

Serotonin and GABA are the main transmitters that play a role in the formation of NREM sleep. Acetylcholine, glutamic acid, and aspartic acid are involved in the neurochemical mechanisms of REM sleep. M. Jouvet hypothesized that REM sleep depends on the "triggering" serotoninergic mechanisms, while noradrenergic and cholinergic processes play a secondary role in this state [41].

In whole, these data illustrate the similarity of various neurochemical mechanisms for emotional states and sleep.

\section{Conclusion}

The results of studies performed by Russian and foreign researchers allow us to make the following principal conclusions on a relationship between the emotional state and sleep.

1. The states of wakefulness and sleep are the interrelated functional systems, which provide an adaptive and resultant activity of the body.

2. The sleep-wakefulness cycle is characterized by a close relationship between the emotional state of an individual during wakefulness and type of nocturnal sleep.

3. Various phases of nocturnal sleep have a different contribution to the sleep-wakefulness cycle.

4. A relationship exists between the individual, personal features of a healthy subject and characteristics of nocturnal sleep.

5. The mechanisms of emotional states and sleep are interrelated with each other and characterized by reciprocal relationships.

Due to the existence of common neurophysiological mechanisms and antagonistic relations of neurochemical processes, emotional stress and sleep are "tied in a tight knot". The goal of modern medicine is to solve this knot and to restore a normal physiological sleep in people suffering from emotional stress. Probably, a new class of pharmaceuticals with the complex, selective neurochemical effects to prevent stress and to activate the natural mechanisms of sleep will be developed in the future. High efficacy of these products can be achieved by an individual selection of the ratio between various biologically active substances and objective monitoring of the quality of sleep and its phase structure in a real everyday life.

\section{Acknowledgements}

This work was supported by the Russian Humanitarian Science Foundation (project No. 16-06-00810, 2016-2018; "Psychophysiological Study of the Correlation between Emotional Strain and Structure of a Normal Nocturnal Sleep in Students with Different Academic Performance").

\section{References}

1. Anokhin PK (1965) Emotional strain as a precondition to the development of neurogenic diseases of the cardiovascular system. Annals of the Academy of Medical Sciences of the USSR 20: 10-18.

2. Sudakov KV (1981) Systemic mechanisms of emotional stress. Medicine. Moscow pp: 229.

3. Sudakov KV, Yumatov EA (1991) Emotional stress in modern life. Soyuzmedinform, Moscow p. 81.

4. Chazov El (1975) Emotional stresses and cardiovascular diseases. Annals of the Academy of Medical Sciences of the USSR 8: 3-8. 
5. Yumatov EA (2011) Emotional stress (Volume 1, Chapter 3, 71). Practical aspects of study and prophylaxis of emotional stress (Volume 1, Chapter 4, 103). Social and economic preconditions for the development of emotional stress (Volume 1, Chapter 5, 133). In: Kekelidze, Z.I., (ed.), Manual: Psychiatry of emergency situations. (2ndedn), Revised and enlarged, in two volumes. Regional Psychiatric Hospital of the Ministry of Health of the Khabarovsk Krai.

6. Vein AM, Hecht K (1989) Human sleep. Physiology and pathology. Medicine, Moscow p. 272.

7. Dorokhov VB (2013) Somnology and safety of professional activity. I. P. Pavlov Journal of Higher Nervous Activity 63: 33-47.

8. Romanov Al, Reshetnyak VK (2003) Sleep and its disorders. Mironov, S.P. (Academician of the Russian Academy of Sciences), (ed.), Word, Moscow p. 272.

9. Selye H (1979) Stress without distress. Progress, Moscow p. 123.

10. Sokolov El, Belova EV (1983) Emotions and heart pathology. Science, Moscow p. 301.

11. Yumatov EA (1997) In: Sudakov, K.V. and Petrov, V.I., (eds.), Emotional stress: Theoretical and clinical aspects, Committee for Press and Information, Volgograd p: 168 (in Russian).

12. Yumatov EA (1980) Cardiovascular functions in emotional loads. Human Physiology 5: 893-906.

13. Yumatov EA (2009) Dynamic organization of emotions and emotional stress. In: Proceeding of the Sixth Simonov Readings, Russian Physician, Moscow 48: 13-46.

14. Valdman AV (1972) Experimental neurophysiology of emotions. Medicine, Leningrad 271.

15. Airapetyants MG (1985) Experimental neuroses. Advances in Physiological Sciences 16: 8-32.

16. Vein AM, Sudakov KV, Levin Ya I, Yumatov EA, Kovrov GV, et al. (2001) Stages of sleep after psychoemotional exposures: Individuality of variations. I. M. Sechenov Physiological Journal 87: 289-295.

17. Kovalzon VM (2011) Bases of somnology. Physiology and neurochemistry of the sleep-wakefulness cycle. Binom. Laboratory of Knowledge, Moscow.

18. Clemente CD, Sterman MB, Wyrwicka W (1963) Forebrain inhibitory mechanisms conditioning of basal forebrain induced E.E.G. synchronization and sleep. Exptl Neurol 7: 404-417.

19. Everson CA, Bergmann BM, Rechtschaffen A (1989) Sleep deprivation in the rat: III. Total sleep deprivation. Sleep 12: 1321.

20. Harrison $Y$, Horne JA (2000) The impact of sleep deprivation on decision making: a review. J Exp Psychol Appl 6: 236-249.

21. Sudakov KV, Yumatov EA, Tarakanov OP (1995) Cross-correlation autonomic criterion of emotional stress. Human Physiology 23: 87-95.

22. Yumatov EA, Pertsov SS, Dudnik EN, Kramm MN, Strelkov NO (2014) Information complex for a systemic monitoring of sleep under everyday conditions. Biomedical Radioelectronics 3: 58-64.

23. Yumatov EA, Pertsov SS, Dudnik EN, Kramm MN, Strelkov NO (2015) Monitoring of human physiological functions during everyday nocturnal sleep. Pathophysiology and Experimental Therapy 59: 47-54.
24. Simonov PV (1981) Emotional brain. Science, Moscow 216.

25. Jasper H (1949) Diffuse projection system. EEG clin. Neurophysiol 1: 405-419.

26. Moruzzi G (1966) The functional significance of sleep with particular regard to the brain mechanisms underlying consciousness. In: Brain and conscious experience. J.C. Eccles (ed.) Berlin-Heidelberg, New York.

27. Moruzzi G (1962) The sleep-waking cycle. Ergebn Physiol 64: 2-164.

28. Oswald I (1962) Sleeping and waking. Amsterdam, Elsevier $\mathrm{p}$ : 232.

29. Rossi GF (1963) Sleep-inducing mechanims in the brain stem. Electroencephalog Clin Neurophysiol Suppl 24: 113-132.

30. Rossi GF, Favale E, Hara T, Givssani A, Sacco G (1961) Researches on the nervous mechanism underlying deep sleep in the cat. Arch Ital Biol 99: 270-292.

31. Rossi GF, Minobe K, Candia O (1963) An experimental study of the hypnogenic mechanisms of the brain stem. Arch Ital Biol 101: 470492.

32. Hess W (1929) The mechanisms of sleep. Amer J Psychol 90: 386-387.

33. Tokizane T (1965) Hypothalamic control of cortical activity and some observations during different states of sleep. In: Aspects anatomo-fonctionnels de la physiologie du sommeil. Ed. by M. Jouvet. Paris Centre Natl Rech Sci p: 15I -184.

34. Clemente CD, Sterman MB, Wyrwicka W (1963) Forebrain inhibitory mechanisms conditioning of basal forebrain induced E.E.G. synchronization and sleep. Exptl Neurol p. 404-417.

35. Koella WP (1984) The organization and regulation of sleep. Experientia 40: 309-338.

36. Pigarev IN (2013) Visceral theory of sleep. I. P. Pavlov Journal of Higher Nervous Activity 63: 86-104.

37. Jouvet M (1962) Sur l'existence d'un systeme hypnique pontolimbique. Ses rapports avec l'activite onirique. In Physiologie de I'hipocampe. P. Passouant (ed.) Paris. Centre Natl Rech Sci p: 297-330.

38. Jouvet M (1962) Recherches sur les structures nerveuses et les mecansimes responsables des differentes phases du sommeil physiologique. Arch Ital Biol 100: 125-206.

39. Jouvet M (1965) Etude de la dualité des états de sommeil et des mécanismes de la phase paradoxale. Aspects anatomofonctionnels de la physiologie du sommeil. M. Jouvet. (ed.) Paris. Centre National de la Recherche Scientifique p. 393-442.

40. Jouvet M (1967) Neurophysiology of the states of sleep. Physiologycal Reviews 47: 117-177.

41. Jouvet $M$ (1988) The regulation of paradoxical sleep by the hypothalamo-hypophysis. Arch Ital Biol 126: 259-274.

42. Val'dman AV (1987) Molecular-and-biological processes in the dynamics of emotional and stress reactions. Annals of the Academy of Medical Sciences of the USSR 6: 11-15.

43. Sudakov KV (1989) Oligopeptides in the mechanisms for resistance to emotional stress. Pathological Physiology 1: 3-11.

44. Yumatov EA (1992) Prolactin in the mechanisms for resistance to emotional stress. In: Psychoemotional stress. Vol. 1, P. K. Anokhin (ed.) Institute of Normal Physiology, Russian Academy of Medical Sciences, Moscow pp. 57-71. 
45. Holmes CJ, Jones BE (1994) Importance of cholinergic, GABAergic, serotoninergic and other neurons in the medial medullary reticular formation for sleep-wake states by cytotoxic lesion in cat. Neuroscience 62: 1179-1200.

46. Matsumoto J, Jouvet M (1964) Effets de la Reserpine, DOPA et 5 HTP sur les deux etats de sommeil. Compt Rend Soc Biol 158: 2135-2139.

47. Tononi G, Cirelli C (2006) Sleep function and synaptic homeostasis. Sleep Med Rev 10: 49-62.

48. Jouvet M, Pujol JF (1974) Effects of central alterations of serotonergic neurons upon the sleep-waking cycle. In: Serotonin - New Vistas, Advances in biochemical pharmacology. Costa, E., Gessa, G. L. \& Sandler, M. (eds.) New York, Raven 11: 199-209.

49. Dahlstrom A, Fuxe K (1964) Evidence for the existence of monoamines containing neurons in the central nervous system. Acta Physiol Scand 62: 232.

50. Hobson JA (1964) The effect of LSD on the sleep cycle of the cat. Electroencephalog Clin Neurophysiol 17: 52-56.

51. Wurtman RJ, Axelrod J, Chu EW (1963) Melatonin, a pineal substance: effect on the rat ovary. Science 141: 277-278.

52. Wurtman RJ (1985) Melatonin as a hormone in humans: A history. Yale J Biol Med 58: 547-552.
53. Hernandez-Peon RA (1965) A cholinergic hypnogenic limbic forebrain-hindbrain circuit. In, Aspects anatomo-fonctionnels de la physiologie du sommeil. M. Jouvet. (ed.) Paris Centre Natl Rech Sci pp. 63-84.

54. Hernandez-Peon RA, Chavez-Ibarra G, Morgane PJ, Timo-lara C (1963) Limbic cholinergic pathways involved in sleep and emotional behavior. Exptl Neurol 8: 93-111.

55. Hernandez-Peon R, O'Flaherty I, Mazzuchelli-O'Flaherty A (1967) Sleep and other behavioral effects induced by acetylcholine stimulation of basal temporal cortex and striate structures. Brain Res 4: 243-267.

56. Matsuzaki M, Takagi H, Tokizane T (1964) Paradoxical phase of sleep: its artificial induction in the cat by sodium butyrate. Science 146: 1328-1330.

57. Onoe H (1998) Molecular and neuroanatomical mechanisms of sleep-wakefulness regulation by prostaglandins D2 and E2. Nippon Yakurigaku Zasshi 112: 343-349.

58. Hayaishi O (1991) Molecular mechanisms of sleep-wake regulation: Roles of prostaglandins D2 and E2. FASEB J 5: 2575-2581. 